5. Cummings SR, Black DM, Thompson DE, et al Effect of alendronate on risk of fracture in women with low bone density but without vertebral fractures: results from the Fracture Intervention Trial. JAMA 1998;28:2077-82

CMAJ 2011. DOI:10.1503/cmaj.111-2032

\section{Intention-to-treat and per-protocol analysis}

I congratulate $C M A J$ and Boutis and colleagues for a brilliant research paper. ${ }^{1}$ Intention-to-treat analysis is a comparison of the treatment groups that includes all patients as originally allocated after randomization. This is the recommended method in superiority trials to avoid any bias. For missing observations, "last value carried forward" is the recommended method.

Per-protocol analysis is a comparison of treatment groups that includes only those patients who completed the treatment originally allocated. If done alone, this analysis leads to bias.

In noninferiority trials, both intention to treat and per-protocol analysis are recommended; both approaches should support noninferiority. In the article by Boutin and colleagues, intention to treat should have included 50 patients in either group as per randomization or at least 45 in the group with splints (in 4 patients, the diagnosis was wrong) and 50 in the group with casts; this may change the results to indicate a borderline effect. In that article, the analysis was done with 43 patients in the splint group and 49 in the cast group, which appears to be a per-protocol analysis, though it was called an intention-to-treat analysis. Hence, noninferiority can be concluded only after analysis by both approaches.

\section{Pankaj B. Shah}

Associate professor, Department of

Community Medicine, SRMC \& RI, SRU,

Chennai, India

\section{Reference}

1. Boutis K, Willan A, Babyn P, et al. Cast versus splint in children with minimally angulated fractures of the distal radius: a randomized controlled trial. CMAJ 2010;182:1507-12.

CMAJ 2011. DOI:10.1503/cmaj.111-2033

We thank Dr. Shah for his thoughtful comments on our article. ${ }^{1}$ As he pointed out, 50 patients were initially randomized to each treatment arm. The four patients randomized to the splint group for whom there were diagnostic errors had to be excluded immediately for safety reasons because the fractures required a different treatment strategy. Some experts would advocate continuation of such patients in the trial, while others would agree that patients enrolled in error should be excluded. We adopted the latter approach because all errors in enrolment were related to diagnostic mistakes that were revealed within 24 hours after randomization.

Among the remaining 46 patients in the splint group and 50 in the cast group, we did not have any primary outcome data for 4 (3 splint, 1 cast) because they were lost to follow up for this outcome. We chose not to account for missing data beause it was such a small number of patients and unlikely to affect the outcome. However, Dr. Shah raises a valid point. We conducted the analysis again, giving the missing cast patient the highest possible score of 100 and the three missing splint patients the lowest observed score in their group (73.28). The lower limit of $90 \%$ confidence interval was then -3.37 and the $p$ value $<0.0001$, thereby rejecting the null hypothesis that the splint is worse than the cast by more than 7 points. These results support the original findings in our article.

\section{Kathy Boutis MD}

Department of Pediatrics, The Hospital for Sick Children, University of Toronto

\section{Andrew Willan PhD}

Sickkids Research Institute

Toronto, Ont.

\section{Reference}

1. Boutis K, Willan A, Babyn P, et al. Cast versus splint in children with minimally angulated fractures of the distal radius: a randomized controlled trial. CMAJ 2010;182:1507-12.

CMAJ 2011. DOI:10.1503/cmaj.111-2034

\section{Electronic medical records: small can be idiosyncratic}

In the article referring to Canada Health Infoway needing a watchdog, ${ }^{1}$ a few things should be considered. Thirty years ago, I was involved with a grassroots approach to try to build an elec- tronic medical record (EMR). It soon became clear that to develop a highperformance product was going to take a multimillion-dollar effort. I have since seen one attempt after another to do the same thing; typically the products are incomplete, use impoverished information models and have idiosyncratic functionality. The problem is that physicians keep buying these products.

In one instance, my office accumulated about 800000 lab results over a 10 -year period. When we changed to a different EMR vendor, the identifier for each test was wiped out because the provincial requirement did not include that identifier. The problem was that the needs of the end user were not being met by the laboratories using legacy systems, and that those who set the provincial requirements did not not recognize this as an issue.

The requirements for a high-performance EMR are not immediately apparent to typical clinical users. They don't seem to be apparent to some EMR vendors who listen to those typical users. There seems to be a widespread awareness of the tremendous amount of work that has been done to determine EMR requirements. If this work was actually considered, we could have made an impressive leap forward in EMR functionality. However, there are so few people interested that there is no critical mass to perform the work on a small scale; a national effort is required.

Although there could be improvements at Infoway, I think the larger problem is that owners and funders of outdated legacy systems are not willing to move forward with systems that would be more conducive to delivering what is needed today.

Raymond Simkus MD

Brookswood Family Practice, Langley, BC

\section{Reference}

1. Webster PC. Experts call for health infoway "watchdog." CMAJ 2011;183:298-299.

CMAJ 2011. DOI:10.1503/cmaj.111-2035

Some letters have been abbreviated for print. See www.cmaj.ca for full versions. 